\title{
La educación de género: más allá de la asistencia y la escolarización
}

\author{
Romelia Hinojosa Luján \\ María Silvia Aguirre Lares \\ Profesoras investigadoras \\ Secretaría de Educación, Cultura y Deporte del Estado \\ de Chihuahua \\ Servicios Educativos del Estado de Chihuahua
}

\section{Resumen}

$\mathrm{E}$

ste artículo presenta un comparativo de diagnósticos realizados en cinco municipios del estado de Chihuahua. El propósito general era buscar brechas de género a través de cinco categorías (población, entorno familiar, educación, trabajo, participación política y salud), sin embargo este texto se focaliza en la educación de las mujeres. El diagnóstico se realiza a través de fuentes consideradas cuantitativas, sin embargo, se enriquece con las interpretaciones de las informantes obtenidas a través de entrevistas y grupos focales, lo que reafirma el enfoque participativo de la investigación. La tesis que se defiende es que aunque la mujer en la última época presenta mayores cifras de asistencia escolar y un mayor grado de escolaridad que los hombres, persisten estereo- tipos tradicionales de género, aprendidos y reforzados socialmente. Ante este panorama, las acciones compensatorias se deben dirigir a unos y a otras: sea para elevar los índices de escolaridad, pero sobre todo, para transformar la cultura de género que prevalece en el imaginario social.

\section{Palabras clave}

Estudio de género, educación, mujeres, escolarización

\section{Introducción}

Se comprende que un diagnóstico es clave para tomar mejores decisiones, además permite identificar aspectos que regularmente no son reconocidos por el imaginario social. Para estos diagnósticos, fue imprescindible recurrir a la perspectiva de género considerada una "herramienta conceptual que busca 
mostrar que las diferencias entre hombres y mujeres, se dan no solo por la determinación biológica, sino por las diferencias culturales asignadas a los seres humanos"(INMUJERES, 2007: 104).

Gracias al aporte de esta perspectiva se ha ampliado la posibilidad de estudiar y generar información relativa a la condición de las mujeres como punto de partida para elaborar políticas públicas en las que se institucionalice la equidad de género.

Por lo regular, los datos que se analizan en los diagnósticos tienen un sustento en la representatividad y la generalización de la población estudiada. Una característica de este tipo de estudios, es que no se ocupan de los pensamientos y creencias que orientan las acciones de hombres y mujeres. Para superar esta postura, los estudios de género promueven metodologías participativas donde los sujetos no sólo se expresen sobre su condición de género, sino que planteen formas de superar esta condición. Por ello los diagnósticos que aquí se presentan, no se limitaron a proporcionar datos estadísticos por la condición de sexo, aún cuando estos resulten imprescindibles, sino que trasciende hacia las dimensiones cualitativas o subjetivas de las relaciones de poder entre los géneros.

En el año 2009 realizamos cinco diagnósticos para estudiar la condición de las mujeres en los municipios chihuahuenses de: Hidalgo del Parral, Rosales, Camargo, Coyame del Sotol y Rosario. Los tres primeros urbanos, los dos últimos del ámbito rural puesto que su población no rebasa los 2,500 habitantes.

Aunque cada uno de los diagnósticos municipales estuvo organizado en las categorías de Población, Familia, Educación, Trabajo y
Participación Política, esta ponencia da cuenta de los hallazgos realizados únicamente sobre la condición educativa de las mujeres y las expectativas que se tienen sobre ella, en un ejercicio comparativo entre los cinco municipios.

En el caso de nuestro país, son de reciente cultivo los estudios de corte estadístico que indagan sobre la condición de las mujeres y los estudios interpretativos tienen aún menos presencia histórica. No es fortuita la atención que el Estado mexicano ha puesto en el estudio de las mujeres, más bien es resultado de una larga lucha feminista que permanentemente ha buscado, por diferentes vías, la visibilización y la denuncia de las situaciones de discriminación y desigualdad de que son objeto las mujeres, aún hoy, en pleno siglo XXI.

Los antecedentes recientes sobre diagnósticos, como el que nos ocupa, se ubican en los estudios realizados por el Instituto Nacional de Estadística, Geografía e Informática (INEGI) y por el propio Instituto Nacional de las Mujeres (INMUJERES). Entre los productos que ofrecen información de alcance nacional, se cuentan estudios sobre violencia hacia la mujer, su condición sociodemográfica y socioeconómica, el uso del tiempo libre, la situación de las indígenas, estudios de mujeres migrantes, entre otros temas. Para el caso de Chihuahua, el Instituto Chihuahuense de la Mujer también ha realizado encuestas en el contexto estatal, para tener un panorama de la situación femenina sobre algunos rubros como: violencia (ICHMUJERES, 2007b), trata de mujeres (ICHMUJERES, 2007a), percepciones masculinas sobre las mujeres (ICHMUJERES, 2008a), brechas de género y mujeres migrantes (ICHMUJERES, 2008b). 
En el contexto de los municipios, son esporádicos los casos de diagnósticos referidos a este tipo de temas. Se encuentran entre ellos, el realizado en Delicias y en Bocoyna en 2008 y otro sobre la población femenina de Juárez. Por ello, el trabajo aquí presentado puede ser considerado pionero en su ramo, máxime cuando ofrece la posibilidad de comparación entre municipios rurales y urbanos.

\section{Objetivos}

Obtener información de opiniones y creencias, relacionadas con la situación educativa de las mujeres que señale rutas de acción para la definición de la política educativa.

\section{Preguntas}

¿Cuál es la situación educativa de las mujeres en los municipios?

¿Cuáles son los niveles de escolarización de la población femenina en cada uno de los municipios?

¿Cuáles son las necesidades relativas a la condición educativa de las mujeres?

\section{Metodología}

Para elaborar los diagnósticos municipales se utilizó un enfoque participativo con metodología mixta, esto es, se recurrió a técnicas propias de la investigación cuantitativa y de la investigación cualitativa. Los datos esencialmente cuantitativos se recuperaron a través de una encuesta que se aplicó a una muestra representativa de la población de mujeres mayores de quince años. Se realizó una muestra aleatoria de población finita o población conocida de la que se desea conocer la proporción que fue encuestada. Con un nivel de confianza de $95 \%$ y un error del 5\%.
Otra información cuantitativa se recopiló de los sitios oficiales de gobierno dedicados a generar estadísticas desagregadas de los municipios como lo es el INEGI, CONAPO e INMUJERES. El tratamiento cualitativo de la información se recuperó a través de varias técnicas como las entrevistas individuales en las que participaron mujeres del municipio considerando el criterio de la diversidad, esto es, se acudió a jóvenes, casadas, trabajadoras remuneradas, amas de casa, solteras y adultas mayores. Otras fuentes fueron las entrevistas individuales focalizadas a informantes clave de cada uno de los municipios que, por su función atienden o están relacionados con grandes grupos de mujeres. También se realizaron grupos de discusión, a donde acudieron mujeres representativas de la diversidad femenina en los municipios, quienes dialogaron en torno a su situación de género.

Los resultados mostraron las brechas de género relativas al nivel de escolaridad, las expectativas que se tienen sobre la educación formal y las principales problemáticas que las mujeres tienen para acrecentar su nivel de escolaridad.

Por último, a partir de esos informes ofrecidos a los municipios, se realiza este estudio comparativo en el que entran en juego las cifras obtenidas y su contextualización a partir de las realidades diferentes que viven estas personas.

\section{Hallazgos}

La educación de las mujeres ocupa una buena parte de la agenda política. Los esfuerzos se han dirigido, sobre todo, a elevar la cobertura y los niveles de escolarización. Los beneficios esperados se extienden hacia 
mejores condiciones de educabilidad de las mujeres y sus hijos.

Una mujer con un mayor nivel de educación se inclinará a tener pocos hijos (as) y más sanos: ella fomentará el interés por la educación en sus hijos (as), haciendo posible a las futuras generaciones aprender y contribuir de una mejor manera en la sociedad. Una mujer con mayor nivel de educación estará más preparada para ingresar al sistema laboral y contribuir para mantenerse ella y a su familia. En los países en desarrollo la educación de las mujeres y su capacitación para el sistema laboral son una manera eficaz para combatir la pobreza (Gallegos, 2007).

Una breve descripción de los municipios brindará mayor información y un panorama claro sobre lo que se vive en la cotidianeidad. La Tabla 1 nos brinda los elementos más ge- nerales de la población de estos municipios:

El índice de feminidad es el número de mujeres por cada 100 hombres.

El grado de marginación es un índice que considera tres dimensiones estructurales de la marginación: educación, vivienda e ingresos por el trabajo. Identifica ocho formas de exclusión y mide su intensidad como porcentaje de la población que no participa del disfrute de bienes y servicios esenciales para el desarrollo de sus capacidades básicas: porcentaje de personas con 15 años o más analfabetas, porcentaje de personas con 15 años o más sin primaria terminada; porcentaje de viviendas sin agua entubada, porcentaje de viviendas con piso de tierra, porcentaje de viviendas sin sanitario, porcentaje de viviendas con hacinamiento y porcentaje de viviendas sin electricidad.

Tabla 1

\begin{tabular}{|c|c|c|c|c|c|c|c|}
\hline & \multicolumn{2}{|c|}{ Población } & \multirow{2}{*}{$\begin{array}{l}\text { Índice de } \\
\text { femini- } \\
\text { dad }\end{array}$} & \multirow[b]{2}{*}{$\begin{array}{c}\text { Grado de } \\
\text { marginación }\end{array}$} & \multirow[b]{2}{*}{ IDH } & \multirow[b]{2}{*}{ IDG } & \multirow{2}{*}{$\begin{array}{c}\text { Porcentaje de } \\
\text { pérdida en de- } \\
\text { sarrollo humano } \\
\text { por la desigual- } \\
\text { dad }\end{array}$} \\
\hline & Hombres & Mujeres & & & & & \\
\hline Rosario & 1305 & 1270 & 103 & $\begin{array}{c}-.40321 \\
\text { (Bajo) }\end{array}$ & $\begin{array}{c}.690 \\
\text { (Medio alto) }\end{array}$ & $\begin{array}{c}.7241 \\
50 \text { lugar } \\
\text { estatal }\end{array}$ & 4.58 \\
\hline $\begin{array}{c}\text { Coyame del } \\
\text { Sotol }\end{array}$ & 801 & 907 & 88 & $\begin{array}{c}-.77371 \\
\text { (Bajo) }\end{array}$ & $\begin{array}{c}.758 \\
\text { (Medio alto) }\end{array}$ & $\begin{array}{c}.7708 \\
42 \text { Lugar } \\
\text { estatal }\end{array}$ & 3.51 \\
\hline $\begin{array}{c}\text { Hidalgo del } \\
\text { Parral }\end{array}$ & 51,998 & 48,823 & 107 & $\begin{array}{c}-1.81554 \\
\text { (Muy bajo) }\end{array}$ & $\begin{array}{c}.810 \\
\text { (Alto) }\end{array}$ & $\begin{array}{c}.8699 \\
\text { 3er lugar } \\
\text { estatal }\end{array}$ & 1.16 \\
\hline Camargo & 23,247 & 22,605 & 103 & $\begin{array}{c}-1.6126 \\
\text { (Muy bajo) }\end{array}$ & $\begin{array}{c}.796 \\
\text { (Medio alto) }\end{array}$ & $\begin{array}{c}.8400 \\
\text { 8० lugar } \\
\text { estatal }\end{array}$ & 1.36 \\
\hline Rosales & 7,444 & 7,525 & 98 & $\begin{array}{c}-1.02352 \\
\text { (Bajo) }\end{array}$ & $\begin{array}{c}.769 \\
\text { (Medio alto) }\end{array}$ & $\begin{array}{c}.8146 \\
19 \circ \text { lugar } \\
\text { estatal }\end{array}$ & 1.45 \\
\hline
\end{tabular}

Fuentes: INEGI y CONAPO, principalmente 
IDH, Se trata de un indicador compuesto, comparable internacionalmente, que combina: i) la longevidad (medida mediante la esperanza de vida al nacer); ii) el logro educacional (a través de la alfabetización de adultos y la matrícula combinada de varios niveles educativos); y iii) el nivel de vida, mediante el PIB per cápita anual ajustado (paridad del poder adquisitivo en dólares).

IDG, el Índice de Desarrollo ajustado por Género, mide el progreso con las mismas variables básicas que el IDH, pero refleja las desigualdades en términos de progreso entre mujeres y hombres. La metodología utilizada penaliza la desigualdad, de manera tal que el IDG disminuye cuando descienden los niveles de progreso tanto de mujeres como de hombres a nivel nacional o cuando aumentan las disparidades en el progreso. Entre mayores son las diferencias de las capacidades básicas, tanto menor es el IDG de un país en comparación con su IDH. El IDG es simplemente el IDH actualizado o ajustado para reflejar las desigualdades basadas en el género.

Si bien, no estamos hablando de los extremos o colas en cuanto a posicionamientos en los índices, sí podemos establecer claramente dos grupos o tipologías de municipios: aquellos que tienen mayor grado de desarrollo humano y menor desigualdad de género, grupo integrado por Hidalgo del Parral, Rosales y Camargo; y el otro grupo en que el desarrollo humano y la desigual- dad de género es más profunda: Rosario y Coyame.

Como se puede apreciar en las Gráficas 1, 2 y 3 , en las tasas e indicadores educativos, las mujeres aventajan a los hombres; a excepción de la tasa de alfabetismo en Hidalgo del Parral, en la que los hombres superan ligeramente al índice de las mujeres. La cultura patriarcal que convertía a la escuela en el lugar prohibido para las mujeres se ha venido revirtiendo. Las cifras así lo muestran: son más las mujeres que estudian. Son ahora los hombres quienes sufren las implicaciones de género al tener que dedicarse desde temprana edad al ámbito productivo, según lo expresan los datos cualitativos.

Se destaca que el dominio de la matrícula escolar femenina se concentra en el nivel básico y a medida que la pirámide educacional

Gráfica 1

Grado de escolaridad

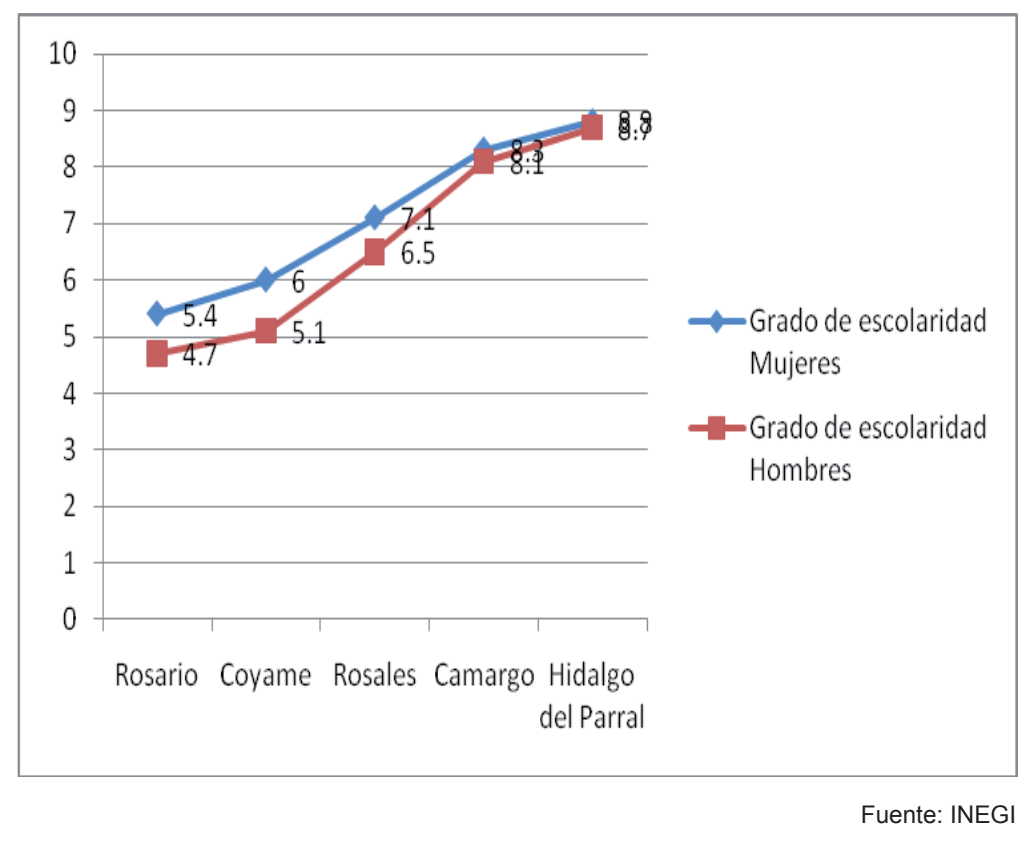


Gráfica 2

Tasa de alfabetismo en la población de 15 años o más

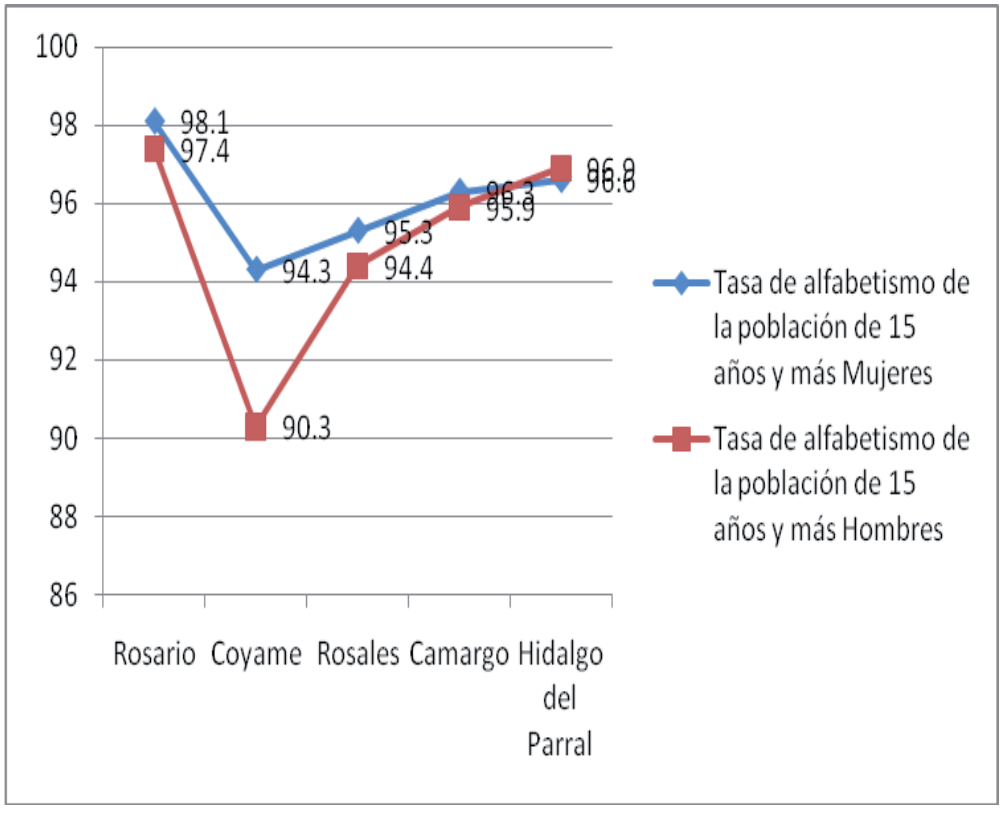

Fuente: INEGI

Gráfica 3

Tasa de asistencia a la escuela
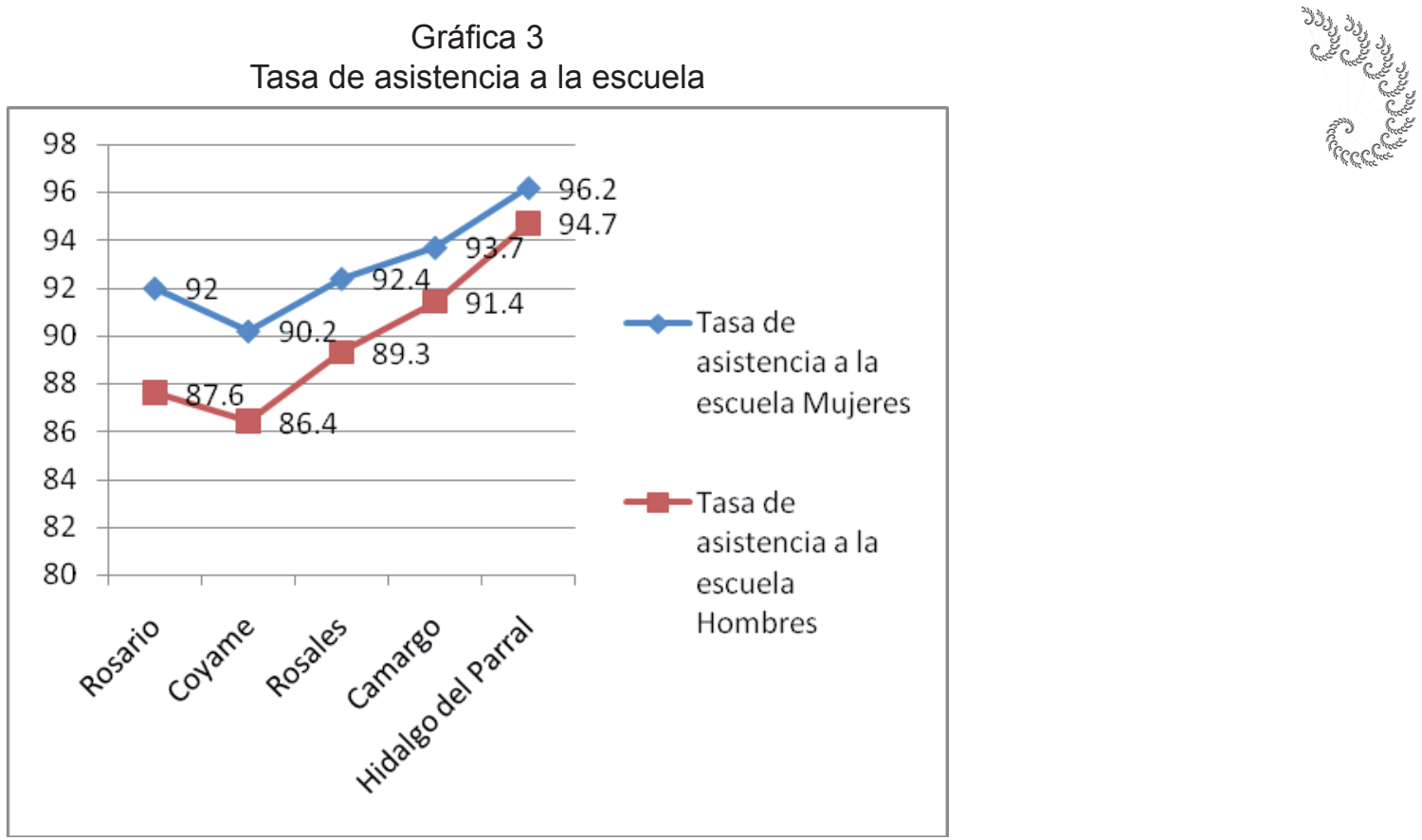

Fuente: INEGI 
asciende a niveles superiores, el fenómeno se revierte. Es decir, ligeramente por arriba de las mujeres están sus parejas en los estudios de profesional terminada y posgrado.

Al comparar los municipios los datos señalan que en los municipios de alto IDG como Hidalgo del Parral, la tendencia educativa de los hombres se concentra más en la educación básica y las mujeres aumentan el grado de escolaridad. Pero el mismo fenómeno se observa en los municipios de bajo IDG donde las mujeres alcanzan un $87 \%$ con estudios de secundaria, mientras sus cónyuges se concentran en la primaria (82\%). Es en los estudios de bachillerato donde se abre una brecha para los dos tipos de municipios: hombres $10 \%$, mujeres $13 \%$, en el caso de Hidalgo del Parral y $11 \%$ y $2 \%$ respectivamente para el municipio de Rosario. Esto se presenta en la Gráfica 4.

Urge entonces hacer un análisis y replanteamiento de la educación hacia los hombres. Se deduce de las creencias de las mujeres que la brecha educativa la sufren ellos por la carga de género al incorporarse tempranamente al trabajo productivo, el caso del municipio rural de Rosario lo explica: allí la agricultura y la ganadería posibilitan el trabajo "informal" de los menores de edad varones.

Sin embargo, si se desea un desarrollo equilibrado, y para que los hombres compartan la necesidad de que la mujer se realice plenamente, es necesario haber avanzado mucho más en la carrera educativa que la escuela primaria.

Gráfica 4

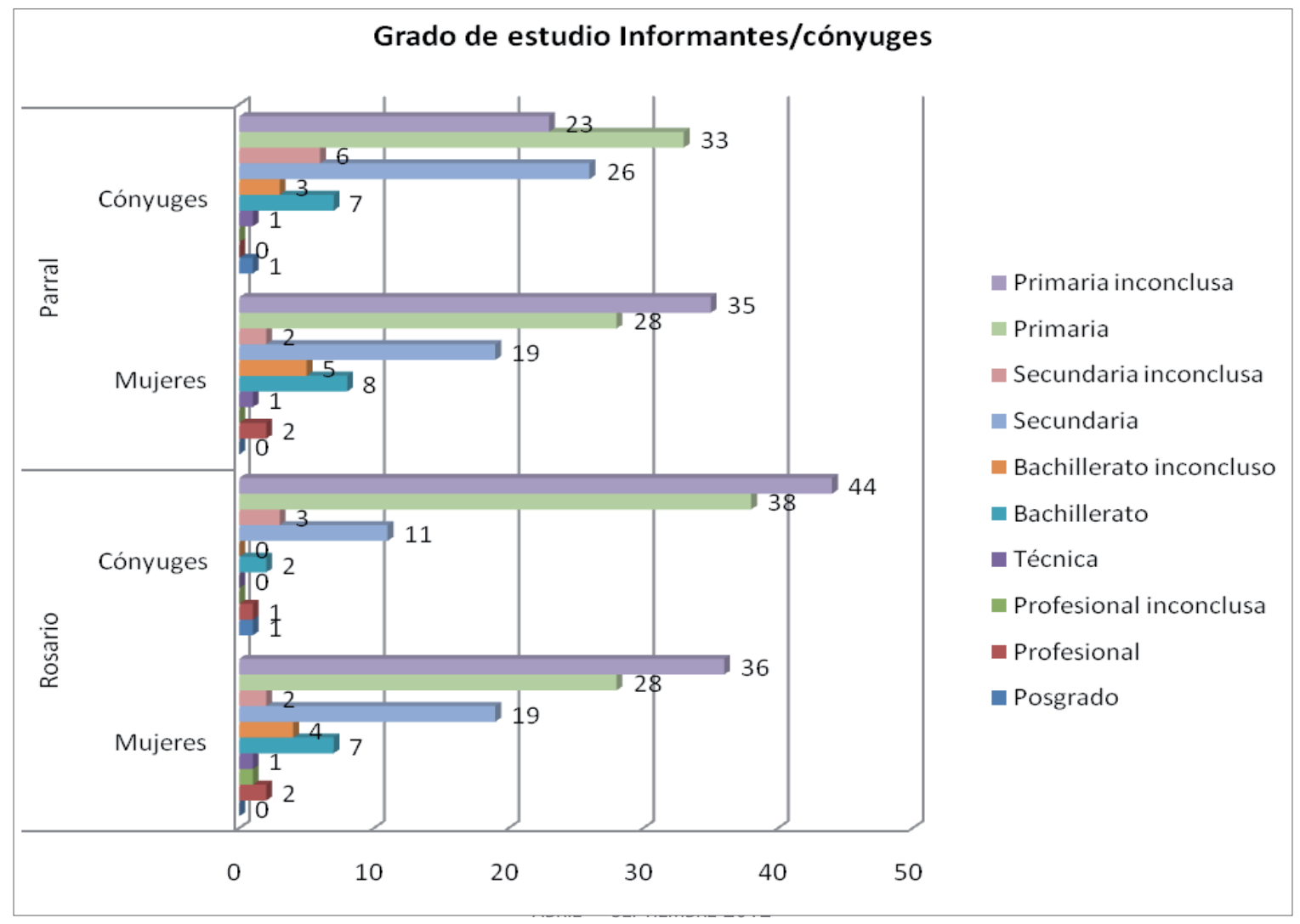


Comparemos ahora, en la Tabla 2, los porcentajes de las causas esgrimidas por las mujeres que les impiden seguir estudiando.

Tabla 2

\begin{tabular}{|l|c|l|c|c|c|c|}
\hline Municipio & $\begin{array}{l}\text { Razones } \\
\text { económicas }\end{array}$ & $\begin{array}{l}\text { Hasta donde } \\
\text { llegó quiere } \\
\text { estudiar }\end{array}$ & $\begin{array}{l}\text { Piensa seguir } \\
\text { estudiando }\end{array}$ & $\begin{array}{l}\text { Hijos o } \\
\text { maternidad }\end{array}$ & $\begin{array}{l}\text { Esposo o } \\
\text { padres no les } \\
\text { permiten }\end{array}$ & $\begin{array}{l}\text { No hay ofertas } \\
\text { educativas en } \\
\text { su localidad }\end{array}$ \\
\hline Rosales & 41.5 & 23 & 5.3 & 26 & 5.9 & 4.8 \\
\hline Camargo & 28 & 21 & 26 & 11 & 8 & 4 \\
\hline $\begin{array}{l}\text { Hidalgo del } \\
\text { Parral }\end{array}$ & 33 & 23 & 18 & 22 & 2 & .4 \\
\hline Rosario & 16 & 33.7 & 9.7 & 10.8 & 2.3 & 24 \\
\hline
\end{tabular}

Destaca de esta información el municipio de Camargo, de alto IDG. Sin embargo las informantes dicen que todavía un $8 \%$ de las mujeres son imposibilitadas a seguir estudiando por falta de "permiso" de los padres o esposo; mientras que el municipio de Rosario que representa al grupo de los municipios de bajo IDG apenas el 2.3\% de las mujeres se siente limitada por el permiso que otorguen los patriarcas de la familia.

Es alarmante cómo en el municipio de Rosales el $41.5 \%$ de las encuestadas asignan a la crisis económica su imposibilidad de continuar estudiando y sólo el 5.3\% de ellas piensa seguir estudiando; del municipio de Rosario destaca cómo el $24 \%$ se siente limitada por la falta de oportunidades escolares en él; así, el problema educativo rebasa el ámbito de acción de la escuela.

En los municipios de bajo desarrollo, uno de los aspectos que las mujeres urgen, es el ofrecimiento de espacios donde se puedan realizar estudios de niveles superiores:

No psss aquí lo que se necesitan son escuelas que den alguna carrera.... apenas tenemos poco que abrió el Сесттесн(bachillerato), pero por eso muchas mujeres se casan, porque no hay más qué hacer (E2B, Rosario, 2009).

En este caso, destaca un 13\% de mujeres que por cargas culturales de género siente limitaciones para continuar su proceso de formación: los embarazos, los permisos de padres y esposos. Las opiniones de las entrevistadas señalan el riesgo de formar uniones o matrimonios a temprana edad:

La mayoría de las jóvenes que terminan la secundaria se casan y no continúan con sus estudios, hay más posibilidades de encontrar un trabajo para las que terminan el bachillerato.... Existen muy pocos aspirantes al nivel superior por problemas económicos ya que tienen que radicar fuera del municipio. El "bachilleres" se abrió hace poco tiempo (E4B, Coyame, 2009).

En este testimonio, que coincide con la encuesta, se destacan las carencias económicas para poder salir a estudiar fuera del municipio.

Veamos ahora las percepciones o preocupaciones que expresan las mujeres en los municipios con más alto rango de desarrollo y menor diferencial de género. Las voces 
tienden más hacia una preocupación de no invadir, a través del estudio, el estatus logrado por los hombres:

Los hombres ven a las mujeres que estudian como que están más "arriba" que ellos (EB5, Parral, 2009).

Los hombres ven bien que las mujeres estudien y ellos también tienen la oportunidad. Y hay otros que no les gusta que las mujeres se superen. Ven a la mujer como una amenaza para ellos (EB3, Parral, 2009).

Aunque también existen preocupaciones afines en ambos tipos de municipios:

No hay ofertas para las muchachas de la preparatoria que no tienen posibilidades (económicas). No hay, en serio... y dice uno: ¿cómo es posible que haya un joven o una señorita con un promedio tan alto que tenga una situación económica muy difícil y ahí se queda? Entonces ¿cómo va a salir de esa pobreza si no hay oportunidades para ella? Esto sí es lamentable y esa chica quería estudiar derecho y aquí en la URN le daban nada más el 30 por ciento (de beca) pero el otro 70 ¿de dónde (lo iba a obtener)? (EA4, Parral, 2009).

\section{Conclusiones}

Se ha demostrado que actualmente las mujeres, ya sean municipios de bajo desarrollo o de alto desarrollo tienen tasas de escolaridad y asistencia mayores a las de los hombres. Este hecho impacta positivamente otras actividades de la mujer: su participación política, la salud, el trabajo productivo y el entorno familiar. Sin embargo, aún cuando existen avances que ahora amenazan a los hombres, existe un fenómeno que se esconde ante la asistencia a la escuela o la escolarización que se recibe: la cultura patriarcal.

Es decir, la escuela no ha podido desmantelar las relaciones sexistas que prevalecen al interior de ella. Asistir y recibir educación, es sinónimo también de estar supeditada al orden y jerarquía patriarcal. No ha sido suficiente aumentar la escolaridad, cuando prevalecen creencias y limitaciones al derecho pleno. Habrá que ver qué tipo de educación se recibe, lo que nos lleva a demandar que la política educativa atienda a la cultura escolar, al currículum oculto. Las creencias señalan que los cambios curriculares y de materiales no han sido suficientes.

El problema es mucho más complejo de lo que la escuela puede intervenir, son sutiles los mecanismos que reproducen la cultura patriarcal y no basta con la capacitación del profesorado. La identidad de género es un aspecto que escapa a la conciencia del ser humano y cuando se "toca", hay un cambio radical en la vida completa del individuo. Pero de lo que sí estamos seguras, es que la escuela se puede convertir en un laboratorio en el que se ensayen nuevas formas de relación entre los géneros en las que el poder sea distribuido en términos equitativos. Esta sería una escuela coeducativa.

Por último, también reconocemos que la labor para desmantelar las relaciones desiguales del género, no es únicamente de la escuela: los medios masivos de comunicación, la iglesia, la familia y otros aparatos ideológicos más, tienen que ser tocados a profundidad. Insistimos, la asistencia a la escuela, no es garantía del cambio cultura patriarcal. 


\section{Referencias bibliográficas}

Gallegos, J. (2007) Implicaciones educativas para la equidad de género: la importancia de fomentar la educación del género femenino.

ICHMujeres (2007a) Primera encuesta sobre la situación de las mujeres chihuahuenses desde la perspectiva masculina, Chihuahua, Méx., Instituto Chihuahuense de la Mujer/Bismark.

ICHMUJEREs (2007b) Primera encuesta sobre las mujeres migrantes en el estado de Chihuahua, Chihuahua, Méx., Instituto Chihuahuense de la Mujer/Bismark.

ICHMUjeres (2008a) Indicadores de brechas en el estado de Chihuahua, Chihuahua, Méx., Instituto Chihuahuense de la Mujer/Bismark.

ICHMUjeres (2008b) Prevalencia de la trata de mujeres y niñas, Chihuahua, Méx., Instituto Chihuahuense de la Mujer /Procesos y análisis estadísticos, S.C.

ICHMUJEREs (2007) Glosario de género, México, INMUJEREs.

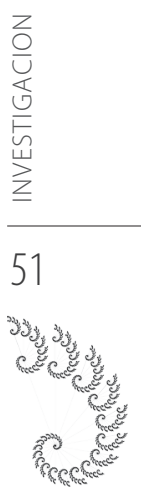

